\title{
DIVERGENCE OF INTERPOLATION POLYNOMIALS IN THE COMPLEX DOMAIN ${ }^{1}$
}

\author{
P. J. O'HARA
}

AbStract. In $1918 \mathrm{~L}$. Fejér gave an example of a function $f(z)$, analytic for $|z|<1$ and continuous for $|z| \leqq 1$, such that the sequence of Lagrange polynomials found by interpolation to $f(z)$ at the roots of unity diverges at a point on the unit circle. More recently S. Ja. $\mathrm{Al}^{\prime}$ per showed that, regardless of how the interpolation points are chosen on the unit circle, a function $g(z)$, analytic for $|z|<1$ and continuous for $|z| \leqq 1$, exists such that the Lagrange polynomials do not converge uniformly to $g(z)$ for $|z| \leqq 1$. In the present paper we present some theory which sheds some light on the results of Fejér and $\mathrm{Al}^{\prime}$ per. A new example of the divergence of Lagrange polynomials is also presented.

1. Introduction. Let $\Gamma$ be an arbitrary Jordan curve in the complex $z$-plane. For each $n, n=0,1, \cdots$ let $S_{n}=\left\{z_{n 0}, z_{n 1}, \cdots, z_{n n}\right\}$ be a set of $n+1$ distinct points on $\Gamma$. If $f(z)$ is a complex-valued function defined on $\Gamma$, let $L_{n}(f ; z)$ denote the polynomial of degree at most $n$ which interpolates to $f(z)$ at the points of $S_{n}$. This polynomial is given by the familiar formula:

$$
L_{n}(f ; z)=\sum_{k=0}^{n} f\left(z_{n k}\right) \lambda_{n k}(z)
$$

where

$$
\begin{aligned}
\lambda_{n k}(z) & =\omega_{n}(z) / \omega_{n}^{\prime}\left(z_{n k}\right)\left(z-z_{n k}\right), \\
\omega_{n}(z) & =\left(z-z_{n 0}\right)\left(z-z_{n 1}\right) \cdots\left(z-z_{n n}\right) .
\end{aligned}
$$

Let $A(\Gamma)$ denote the class of complex-valued functions which are analytic on Int $\Gamma$ and continuous on $\bar{\Gamma}=\Gamma \cup \operatorname{Int} \Gamma$. For the case in which $\Gamma$ is the unit circle, $S_{n}$ is the set of $(n+1)$ st roots of unity, and $f \in A(\Gamma)$, Fejér [8] proved that the sequence $\left\langle L_{n}(f ; z)\right\rangle$ converges to $f(z)$ uniformly on compact subsets of $|z|<1$. This result has been extended to more general curves $\Gamma$ by various authors; see for example Curtiss

Presented to the Society, August 30, 1968 under the title $A$ necessary condition in the theory of interpolation in the complex domain; received by the editors August $3,1969$.

A MS Subject Classifications. Primary 3036, 4110; Secondary 4690.

Key Words and Phrases. Complex Lagrange polynomials, pointwise convergence, uniform convergence, linear functional, uniform boundedness principle.

1 This research was sponsored by the Air Force Office of Scientific Research, Office of Aerospace Research, AF-AFOSR 68-1525. 
[5], [6], [7], $\mathrm{Al}^{\prime}$ per [1], Thompson [15], and O'Hara [12]. With $\Gamma$ the unit circle and $S_{n}$ the roots of unity, Fejér in [8] also exhibited a function $f^{*} \in A(\Gamma)$ such that at a point $z_{0}$ on $\Gamma,\left\langle L_{n}\left(f^{*} ; z_{0}\right)\right\rangle$ is unbounded. Also Al'per [1] showed that no matter how $S_{n}$ is chosen on the unit circle $\Gamma$, there exists $g^{*} \in A(\Gamma)$ such that $L_{n}\left(g^{*} ; z\right)$ does not converge uniformly to $g(z)$ for $|z| \leqq 1$.

In this paper we propose to complement these divergence examples with some theory and further examples. In $\$ 2$ necessary and sufficient conditions are given for the convergence, pointwise and uniform, of $\left\langle L_{n}(f ; z)\right\rangle$ with $f \in A(\Gamma)$. In $\S 3$ these conditions are used to shed some light on the examples of Fejér and $\mathrm{Al}^{\prime}$ per. In the last section, with $\Gamma$ the unit circle, a specific family of functions in $A(\Gamma)$ and sets $S_{n}$ are introduced for which $\left\langle L_{n}(f ; z)\right\rangle$ is unbounded at a point of Int $\Gamma$.

2. Necessary and sufficient conditions for convergence of $\left\langle L_{n}(f ; z)\right\rangle$. Let $\Gamma$ be an arbitrary Jordan curve and suppose $f \in A(\Gamma)$. By referring to the existence of convergent polynomial approximations for $f(z), z$ on $\Gamma$, Curtiss [5, p. 181] remarked that a sufficient condition that $\lim _{n \rightarrow \infty} L_{n}\left(f ; z_{0}\right)=f\left(z_{0}\right)$ for any $z_{0} \in \operatorname{Int} \Gamma$ is that the sequence $\left\langle\lambda_{n}\left(z_{0}\right)\right\rangle_{n=0}^{\infty}$ shall be bounded, where $\lambda_{n}(z)=\sum_{k=0}^{n}\left|\lambda_{n k}(z)\right|, n=0$, $1, \cdots$. The remark remains valid for $z_{0}$ on $\Gamma$. (The convergence is uniform on $\bar{\Gamma}$ if $\left\langle\lambda_{n}(z)\right\rangle$ is uniformly bounded on $\bar{\Gamma}$.) He conjectured that the condition is necessary. We here establish the conjecture by showing that if this sequence is unbounded, then there exist functions $f$ in $A(\Gamma)$ for which $\left\langle L_{n}\left(f ; z_{0}\right)\right\rangle$ is unbounded.

Our starting point is the following simple consequence of a theorem of Rudin [13] (see also [3]).

LEMMA 2.1. Let $\Gamma$ be an arbitrary Jordan curve in the complex z-plane and let $z_{1}, \cdots, z_{m}$ be $m$ distinct points on $\Gamma$. Let $t_{1}, \cdots, t_{m}$ by any $m$ complex numbers (not necessarily distinct). Then there exists $g \in A(\Gamma)$ such that $g\left(z_{j}\right)=t_{j}, j=1, \cdots, m$. Moreover if $\left|t_{j}\right| \leqq 1, j=1, \cdots, m$, then $g$ can be chosen so that $|g(z)| \leqq 1$ for $z \in \bar{\Gamma}$.

We now note that for any fixed $z_{0}$ and for each $n=0,1, \ldots$ the mapping $L_{n}\left(; z_{0}\right): f \rightarrow L_{n}\left(f ; z_{0}\right)$ is a linear functional on the space of bounded complex functions on $\Gamma$ normed with the sup norm. The operator norm $\left\|L_{n}\left(; z_{0}\right)\right\|$ is clearly $\lambda_{n}\left(z_{0}\right)$. There remains a question, here crucial, as to whether this is still the value of $\left\|L_{n}\left(; z_{0}\right)\right\|$ when the domain of $L_{n}\left(; z_{0}\right)$ is restricted to the Banach space $A(\Gamma)$. The answer is affirmative.

Lemma 2.2. The norm $\left\|L_{n}\left(; z_{0}\right)\right\|$ with $L_{n}\left(; z_{0}\right)$ considered as a linear functional on $A(\Gamma)$ is $\lambda_{n}\left(z_{0}\right)$. 
Let $s(z)=|z| / z, z \neq 0, s(0)=1$, and on the points $z_{n k}, k=0, \cdots, n$, define a function $g_{0}$ by $g_{0}\left(z_{n k}\right)=s\left[\lambda_{n k}\left(z_{0}\right)\right]$. Then $\left|g_{0}\left(z_{n k}\right)\right|=1$ and $L_{n}\left(g_{0} ; z_{0}\right)=\lambda_{n}\left(z_{0}\right)$. Now by Lemma 2.1 , there exists a function $g(z)$ $\in A(\Gamma)$ such that $g\left(z_{n k}\right)=g_{0}\left(z_{n k}\right), k=0, \cdots, n$, and $\sup _{z \in \bar{\Gamma}}|g(z)|=1$. Thus by definition of the operator norm, $\left\|L_{n}\left(; z_{0}\right)\right\|=\lambda_{n}\left(z_{0}\right)$ even when the domain of $L_{n}\left(; z_{0}\right)$ is restricted to $A(\Gamma)$.

THEOREM 2.1. If the sequence $\left\langle\lambda_{n}\left(z_{0}\right)\right\rangle$ is unbounded then the sequence $\left\langle L_{n}\left(f ; z_{0}\right)\right\rangle$ is unbounded for all f belonging to some dense $G_{\delta}$ set in $A(\Gamma)$.

This follows from the Banach-Steinhaus theorem [14, p. 98] now that the sequence $\left\langle\lambda_{n}\left(z_{0}\right)\right\rangle$ has been identified with the sequence of operator norms $\left\langle\left\|L_{n}\left(; z_{0}\right)\right\|\right\rangle$ with the domain of $L_{n}\left(; z_{0}\right)$ restricted to $A(\Gamma)$.

Theorem 2.1 deals with the pointwise behavior of the sequence $\left\langle L_{n}(f ; z)\right\rangle$. The following theorem is the natural counterpart of Theorem 2.1 for the uniform behavior of this sequence.

THEOREM 2.2. If the sequence $\left\langle\lambda_{n}\right\rangle$ is unbounded, where $\lambda_{n}=\sup _{\boldsymbol{z}} \in \mathrm{\Gamma}$ $\lambda_{n}(z)$, then the sequence $\left\langle L_{n}(f ; z)\right\rangle$ is not uniformly bounded on $\bar{\Gamma}$ for all $f$ belonging to some dense $G_{\delta}$ set in $A(\Gamma)$.

Since the proof is similar to that of Theorem 2.1 , we present only a brief outline and let the reader supply the details. Consider the linear operator $L_{n}: A(\Gamma) \rightarrow A(\Gamma)$ defined by $L_{n}: f \rightarrow L_{n}(f ; z)$. The norm of this operator is given by $\left\|L_{n}\right\|=\sup _{z \in \bar{\Gamma}} \lambda_{n}(z)=\lambda_{n}$ (the last equality follows from the theory of subharmonic functions). An application of the Banach-Steinhaus theorem completes the proof.

3. Remarks on the examples of Fejér and $\mathrm{Al}^{\prime}$ per. In order to examine the example of Fejér, we begin with the following

Lemm 3.1. If $\Gamma$ is the unit circle and $S_{n}$ is the set of $(n+1)$ st roots of unity, then the sequence $\left\langle\lambda_{n}\left(z_{0}\right)\right\rangle$ is unbounded at each point $z_{0}$ on $\Gamma$ with the exception of $z_{0}=1$.

For in this case $\omega_{n-1}(z)=z^{n}-1$, and hence

$$
\lambda_{n-1}\left(z_{0}\right)=\frac{\left|z_{0}^{n}-1\right|}{n} \sum_{k=0}^{n-1} \frac{1}{\left|z_{0}-z_{n k}\right|}
$$

where $z_{n k}=\exp (2 \pi i k / n)$. If $z_{0} \neq 1$ then there is a subsequence $\left\langle n_{j}\right\rangle$ of the positive integers and a positive number $\delta$ such that $\left|z_{0}^{n_{i}}-1\right| \geqq \delta$, $j=1,2, \cdots$ Let $z_{0}=\exp (2 \pi i \lambda), 0<\lambda<1$, and let $[x]$ denote the largest integer $\leqq x$. Now apply the identity $\exp (i \theta)-\exp (i \alpha)=$ $2 i \sin ((\theta-\alpha) / 2) \exp (i(\theta+\alpha) / 2)$, to obtain for $j$ sufficiently large: 


$$
\begin{aligned}
\lambda_{n_{j-1}}\left(z_{0}\right) & \geqq \frac{\delta}{2 n_{j}} \sum_{k=0}^{n_{j}-1} \frac{1}{\left|\sin \left(\pi \lambda-\pi k / n_{j}\right)\right|} \geqq \frac{\delta}{2 \pi n_{j}} \sum_{k=0}^{\left[n_{j}\right]} \frac{1}{\lambda-k / n_{j}} \\
& \geqq \frac{\delta}{2 \pi} \int_{\lambda-\left[n_{j} \lambda\right] / n_{j}}^{\lambda} \frac{1}{x} d x \geqq \frac{\delta}{2 \pi} \int_{1 / n_{j}}^{\lambda} \frac{1}{x} d x=\frac{\delta}{2 \pi} \log n_{j} \lambda .
\end{aligned}
$$

Fejér's divergence example can now be viewed as a specific realization of the following theorem:

TheOREM 3.1. Let $\Gamma$ be the unit circle and let $S_{n}$ be the set of $(n+1) s t$ roots of unity. Let $T$ be any countable subset of $\Gamma-\{1\}$. Then there is a dense $G_{\delta}$ set in $A(\Gamma)$ such that whenever $f$ belongs to this set, the sequence $\left\langle L_{n}(f ; z)\right\rangle$ is unbounded at each $z \in T$.

Let $T=\left\{z_{1}, z_{2}, \cdots\right\}$ and let $A_{j}$ be the set of $f \in A(\Gamma)$ such that $\sup _{n} L_{n}\left(f ; z_{j}\right)=\infty$. Then by Theorem 2.1 and the lemma above, each $A_{j}$ contains a dense $G_{\delta}$ set in $A(\Gamma)$. Therefore by Baire's category theorem $\bigcap_{j=1}^{\infty} A_{j}$ contains a dense $G_{\delta}$ set in $A(\Gamma)$.

As usual in polynomial approximation theory, relatively light further restrictions on functions in $A(\Gamma)$ may exclude them from the $G_{\delta}$ set of divergence referred to in Theorem 3.1. For example, with $\Gamma$ and $S_{n}$ as in the preceding theorem, it is proved in [7] that when $f \in A(\Gamma)$ is of bounded variation on $\Gamma$, then $\lim _{n \rightarrow \infty} L_{n}(f ; z)=f(z)$ uniformly on $\bar{\Gamma}$. (See also [1, Theorem 2].) We also remark that the results of [4] can be used to extend Lemma 3.1 (and hence Theorem 3.1) to a more general type of Jordan curve $\Gamma$. In this extension the interpolation points $S_{n}$ are the so-called Fejér points on $\Gamma$ (see for example [7]).

We turn now to examine $\mathrm{Al}^{\prime}$ per's result, for which purpose the following lemma is needed.

Lemma 3.2. For any choice of $S_{n}, n=0,1, \cdots$, on the unit circle $\Gamma$, the sequence $\left\langle\lambda_{n}\right\rangle$ is unbounded.

$\mathrm{Al}^{\prime}$ per [1] mentions that this lemma can be proved by slightly modif ying the proof of a similar theorem due to Faber and Bernštein (see $[11$, p. 370]). We shall outline here a different proof. Choose $f \in A(\Gamma)$ and $z_{0}$ on $\Gamma$ so that $T_{n}\left(z_{0}\right)$ diverges, where $T_{n}(z)$ is the $n$th partial sum of the Taylor series for $f(z)$ (see for example [17, p. 300]). Define $g_{t} \in A(\Gamma)$ by $g_{t}(z)=f(t z)$ and let $G_{n}(t)=L_{n}\left(g_{t} ; z_{0} / t\right)$. Now suppose to the contrary that $\lambda_{n} \leqq M, n=0,1, \cdots$. Then for each $t$ on $\Gamma$, $\left\langle\lambda_{n}\left(z_{0} / t\right)\right\rangle$ is bounded and by Curtiss' remark: $\lim _{n \rightarrow \infty} G_{n}(t)=g_{t}\left(z_{0} / t\right)$ $=f\left(z_{0}\right)$. Moreover $\left\langle G_{n}(t)\right\rangle$ is uniformly bounded by $M\left(\sup _{z \in \Gamma}|f(z)|\right)$. Hence if $\left\langle\lambda_{n}\right\rangle$ is bounded then 


$$
\lim _{n \rightarrow \infty} \frac{1}{2 \pi i} \int_{|t|=1} \frac{G_{n}(t)}{t} d t=\frac{1}{2 \pi i} \int_{|t|=1} \frac{f\left(z_{0}\right)}{t} d t=f\left(z_{0}\right) .
$$

However this is impossible since it can be shown that for each $n=0$, $1, \cdots$ the integral in the left member of (3.1) equals $T_{n}\left(z_{0}\right)$. (A similar result for trigonometric interpolation is proved in [10].)

Applying Theorem 2.2 we can slightly improve $\mathrm{Al}^{\prime}$ per's result as follows:

TheOREM 3.2. For any choice of $S_{n}, n=0,1, \cdots$, on the unit circle $\Gamma$, there is a dense $G_{\delta}$ set in $A(\Gamma)$ such that whenever $f$ belongs to this set, the sequence $\left\langle L_{n}(f ; z)\right\rangle$ is not uniformly bounded on $\bar{\Gamma}$.

An apparently open question in connection with the results of this section is as follows: If $\left\langle S_{n}\right\rangle$ and $\Gamma$ are arbitrary, does there exist $z_{0}$ on $\Gamma$ such that $\left\langle\lambda_{n}\left(z_{0}\right)\right\rangle$ is unbounded? (Bernštern [2] has answered this question in the affirmative when $\Gamma$ is replaced by an interval on the real line.) In fact it is apparently unknown whether or not Lemma 3.2 holds for an arbitrary $\Gamma$.

4. Example of divergence for $z_{0}$ in the interior of the unit disc. Let $\Gamma$ be the unit circle. For $\beta$ irrational, $0<\beta<1$, let $S_{n}^{(\beta)}=\left\{z_{n 0}, \cdots\right.$, $\left.z_{n n}\right\}$ where $z_{n k}=\exp (2 \pi i k / n), 0 \leqq k \leqq n-1$, and $z_{n n}=\exp (2 \pi i \beta)$. Also define a function $f_{\beta} \in A(\Gamma)$ by:

$$
f_{\beta}(z)=(z-\exp (2 \pi i \beta))^{1 / 2}
$$

where

$$
(w)^{1 / 2}=|w|^{1 / 2} \exp (\arg w / 2), \quad 2 \pi \beta \leqq \arg w<2 \pi \beta+2 \pi .
$$

The sequence $\left\langle S_{n}^{(\beta)}\right\rangle$ is uniformly distributed (see $[16$, p. 164]) on $\Gamma$. Therefore by a well-known theorem of Fejér $[8]: \lim _{n \rightarrow \infty} L_{n}(f ; z)=f(z)$ uniformly on $\bar{\Gamma}$, providing $f(z)$ is analytic on $\bar{\Gamma}$. However $\sup _{n} \lambda_{n}\left(z_{0}\right)$ $=\infty$ for every $z_{0} \in \operatorname{Int} \Gamma$ (note that $\lambda_{n n}\left(z_{0}\right)=\left(z_{0}^{n}-1\right) /(\exp (2 \pi i n \beta)-1)$ ), and so for each $z_{0} \in \operatorname{Int} \Gamma$ there are functions $f \in A(\Gamma)$ for which $\left\langle L_{n}\left(f ; z_{0}\right)\right\rangle$ is unbounded. The following theorem shows that in the case $z_{0}=0$, one of these functions is $f_{\beta}$ (at least for some $\beta$ 's).

Theorem 4.1. Let $L_{n}\left(f_{\beta} ; z\right)$ be the Lagrange polynomial which interpolates to $f_{\beta}(z)$ at the points of $S_{n}^{(\beta)}$. Then there is a dense set of $\beta$ 's for which $\left\langle L_{n}\left(f_{\beta} ; 0\right)\right\rangle$ is unbounded.

After some computation, it is found from (1.1) that, with square roots determined as in the definition of $f_{\beta}$ : 


$$
\begin{aligned}
L_{n}\left(f_{\beta} ; 0\right) & =-\frac{e^{i 2 \pi \beta}}{n} \sum_{k=0}^{n-1}\left(z_{n k}-e^{i 2 \pi \beta}\right)^{-1 / 2} \\
& =-\frac{e^{i 2 \pi \beta}}{(2)^{1 / 2} n} \sum_{k=0}^{n-1}\left(\sin \left(\frac{\pi k}{n}-\pi \beta\right) \exp \left(i\left(\frac{\pi k}{n}+\pi \beta+\frac{\pi}{2}\right)\right)\right)^{-1 / 2} .
\end{aligned}
$$

Now let $[n \beta]$ denote the largest integer $\leqq n \beta$. Also let $\psi_{n k}=\pi k / n+\pi \beta$ $+3 \pi / 2$ and $\phi_{n k}=\pi k / n+\pi \beta+\pi / 2$. Then the above equation can be written:

$$
\begin{aligned}
L_{n}\left(f_{\beta} ; 0\right)=-\frac{e^{i 2 \pi \beta}}{(2)^{1 / 2} n}\left[\sum_{k=0}^{[n \beta]}\right. & \left(\sin \left(\pi \beta-\frac{\pi k}{n}\right) \exp \left(i \psi_{n k}\right)\right)^{-1 / 2} \\
& \left.+\sum_{k=[n \beta]+1}^{n-1}\left(\sin \left(\frac{\pi k}{n}-\pi \beta\right) \exp \left(i \phi_{n k}\right)\right)^{-1 / 2}\right] .
\end{aligned}
$$

It follows from the definition of $\psi_{n k}$ and $\phi_{n k}$ that:

$$
\begin{array}{ll}
\pi \beta+3 \pi / 2 \leqq \psi_{n k}<2 \pi \beta+3 \pi / 2 ; & 0 \leqq k \leqq\lfloor n \beta\rfloor, \\
2 \pi \beta+\pi / 2<\phi_{n k}<\pi \beta+3 \pi / 2 ; & {[n \beta]+1 \leqq k \leqq n-1 .}
\end{array}
$$

Since $0<\beta<1$ we also obtain:

$$
\begin{aligned}
& 2 \pi \beta+\pi / 2<\psi_{n k}<2 \pi \beta+3 \pi / 2 ; \quad 0 \leqq k \leqq[n \beta], \\
& 2 \pi \beta+\pi / 2<\phi_{n k}<2 \pi \beta+3 \pi / 2 ; \quad[n \beta]+1 \leqq k \leqq n-1 .
\end{aligned}
$$

Now note that: $-\pi<\pi k / n-\pi \beta<0$ if $0 \leqq k \leqq[n \beta]$, and $0<\pi k / n-\pi \beta$ $<\pi$ if $[n \beta]+1 \leqq k \leqq n-1$. Therefore:

$$
\begin{array}{ll}
\sin (\pi \beta-\pi k / n)>0 ; & 0 \leqq k \leqq[n \beta], \\
\sin \left(\pi^{k} / n-\pi \beta\right)>0 ; & {[n \beta]+1 \leqq k \leqq n-1 .}
\end{array}
$$

By applying (4.2), (4.3), and the definition of the square root used to define $f_{\beta}$, the last expression for $L_{n}\left(f_{\beta} ; 0\right)$ can be rewritten (all square roots from here on will be nonnegative):

$$
L_{n}\left(f_{\beta} ; 0\right)=-\left(e^{i 2 \pi \beta} / 2\right)\left[A_{n}^{(\beta)}-i B_{n}^{(\beta)}\right],
$$

where

$$
A_{n}^{(\beta)}=\sum_{k=0}^{[n \beta]} \frac{\cos \left(\psi_{n k} / 2\right)}{n(\sin (\pi \beta-\pi k / n))^{1 / 2}}+\sum_{k=[n \beta]+1}^{n-1} \frac{\cos \left(\phi_{n k} / 2\right)}{n(\sin (\pi k / n-\pi \beta))^{1 / 2}}
$$

and

$$
B_{n}^{(\beta)}=\sum_{k=0}^{[n \beta]} \frac{\sin \left(\psi_{n k} / 2\right)}{n(\sin (\pi \beta-\pi k / n))^{1 / 2}} \sum_{k=\{n \beta]+1}^{n-1} \frac{\sin \left(\phi_{n k} / 2\right)}{n(\sin (\pi k / n-\pi \beta))^{1 / 2}} .
$$


We now consider the following cases individually: $0<\beta<1 / 4$, $1 / 4<\beta<1 / 2,1 / 2<\beta<3 / 4,3 / 4<\beta<1$. In each case we show that there is a dense set of $\beta^{\prime}$ 's for which either $\left\langle A_{10}^{(\beta)}\right\rangle$ or $\left\langle B_{n}^{(\beta)}\right\rangle$ is unbounded. If $0<\beta<1 / 4$ then by (4.1): $3 \pi / 4<\psi_{n k} / 2<\pi$ if $0 \leqq k \leqq[n \beta]$, and $\pi / 4$ $<\phi_{n k} / 2<7 \pi / 8$ if $[n \beta]+1 \leqq k \leqq n-1$. Thus all the terms of $B_{n}^{(\beta)}$ are positive and so:

$$
\begin{aligned}
B_{n}^{(\beta)} & >\frac{\sin \left(\phi_{n,[n \beta]+1} / 2\right)}{n(\sin ((\pi([n \beta]+1) / n)-\pi \beta))^{1 / 2}} \\
& >\frac{\sin (7 \pi / 8)}{\pi^{1 / 2}(n(1-(n \beta-[n \beta])))^{1 / 2}} .
\end{aligned}
$$

Using standard results in the thoery of continued fractions (see for example [9, Chapter X]) it can be shown there is a dense set of $\beta$ 's such that both the sequences

$$
\langle n(n \beta-[n \beta])\rangle_{n=1}^{\infty} \text { and }\langle n(1-(n \beta-[n \beta]))\rangle_{n=1}^{\infty}
$$

have subsequences which tend to zero. Hence $\left\langle L_{n}\left(f_{\beta} ; 0\right)\right\rangle$ is unbounded for $\beta$ belonging to a dense subset of $(0,1 / 4)$. A similar analysis can be made in the remaining three cases.

\section{REFERENCES}

1. S. Ja. $\mathrm{Al}^{\prime}$ per, On the convergence of Lagrange's interpolational polynomials in the complex domain, Uspehi Mat. Nauk 11 (1956), no. 5 (71), 44-50. (Russian) MR 18, 728.

2. S. N. BernšteǏn, Sur la limitation des valeurs d'un polynôme $P_{n}(x)$ de degré $n$ sur tout un segment par ses valeurs en $(n+1)$ points du segment, Bull. Acad. Sci. URSS 1931, no. $8,1025-1050$.

3. E. Bishop, A general Rudin-Carleson theorem, Proc. Amer. Math. Soc. 13 (1962), 140-143. MR 24 \#A3293.

4. J. H. Curtiss, Riemann sums and the fundamental polynomials of Lagrange interpolation, Duke Math. J. 8 (1941), 525-532. MR 3, 115.

5. - Interpolation with harmonic and complex polynomials to boundary values, J. Math. Mech. 9 (1960), 167-192. MR 22 \#4890.

6. - Polynomial interpolation in points equidistributed on the unit circle, Pacific J. Math. 12 (1962), 863-877. MR 29 \#403.

7. - Convergence of complex Lagrange interpolation polynomials on the locus of the interpolation points, Duke Math. J. 32 (1965), 187-204. MR 35 \#1787.

8. L. Fejér, Interpolation und konforme Abildung, Göttingen Nachr. 1918, 319-331.

9. G. H. Hardy and E. M. Wright, An introduction to the theory of numbers, Clarendon Press, Oxford, 1938; 4th ed., Oxford Univ. Press, London, 1960.

10. J. Marcinkiewicz, Quelques remarques sur l'interpolation, Acta Univ. Szeged $8(1936 / 37), 127-130$.

11. I. P. Natanson, Constructive function theory, GITTL, Moscow, 1949; German transl., Akademie-Verlag, Berlin, 1955; English transl., Ungar, New York, 1964. MR 11, 591; MR 16, 1100; MR 33 \#529a. 
12. P. J. O'Hara, A study of interpolation by complex polynomials, Doctoral Dissertation, University of Miami, Coral Gables, Florida, 1968.

13. W. Rudin, Boundary values of continuous analytic functions, Proc. Amer. Math. Soc. 7 (1956), 808-811. MR 18, 472.

14. - Real and complex analysis, McGraw-Hill, New York, 1966. MR 35 $\# 1420$.

15. M. Thompson, Complex polynomial interpolation to continuous boundary data, Proc. Amer. Math. Soc. 20 (1969), 327-332.

16. J. L. Walsh, Interpolation and approximation by rational functions in the complex domain, 2nd ed., Amer. Math. Soc. Colloq. Publ., vol. 20, Amer. Math. Soc., Providence, R. I., 1956; 4th rev. ed., 1965. MR 36 \#1672b.

17. A. Zygmund, Trigonometric series, Vol. I, 2nd rev. ed., Cambridge Univ. Press, New York, 1959. MR 21 \#6498.

Florida Technological University, OrLando, Florida 32816 